\title{
Congenital self-healing reticulohistiocytosis in neonate - a single lesion presentation
}

\begin{abstract}
Congenital Langherhans cell histiocytosis ( $\mathrm{LCH})$ is a rare immune disorder, presenting during the neonatal period with variable cutaneous finding and sometimes as disseminated disease. We describe a case of LCH in large for gestational age (LGA) term male neonate, who presented with the single face skin lesion.
\end{abstract}

Keywords: congenital langherhans cell histiocytosis, skin lesions, congenital selfhealing reticulohistiocytosis

\author{
Volume 8 Issue 6 - 2018
}

\author{
Babatseva E,' Mitsiakos G,' Anastasiadis \\ $\mathrm{K},{ }^{2}$ Kepertis C, ${ }^{2}$ Papacharalampous E,' \\ Karagianni P,' Voziki E,' Kyriakeli G,' Soubasi \\ $\mathrm{V}^{\prime}$ \\ 'Department of Neonatology, Aristotle University of \\ Thessaloniki, Greece \\ ${ }^{2}$ Pediatric Surgery Department Aristotle University of \\ Thessaloniki, Greece
Correspondence: Dr Kleanthis Anastasiadis, 2nd Department of Pediatric Surgery Aristotle University of Thessaloniki, General Hospital "PAPAGEORGIOU”,Thessaloniki, Greece,Tel 003023।3323594, Fax 003023।3323546, Email kaanastesiadis।@gmail.com

Received: August 14, 2018 | Published: December 10, 2018
Abbreviations: LCH, Langherhans cell histiocytosis; LGA, large for gestational age; CSHRH, congenital selfhealing reticulohistiocytosis; CSHR, Congenital selfhealingreticulohistiocytosis;

\section{Introduction}

Langerhans cell histiocytosis ( $\mathrm{LCH})$ is a proliferative disease of macrophage and dendrite cells. ${ }^{1}$ A separate clinical entity termed congenital self-healing reticulohistiocytosis (CSHRH), also known as Hashimoto- Pritzker disease ${ }^{2,3}$ is characterized by skin lesions in the newborn period as a single system cutaneous presentation, in an otherwise healthy infant. The skin lesions spontaneously involutes within weeks to a few months. ${ }^{3,4}$

\section{Case report}

A LGA male neonate was born to a fourth parous 39year-old mother at 39 and 5/7weeks gestation via a vaginal delivery. Immediately after birth he was noted to have a solitary skin lesion-brown, crusted papule on left cheek (Figure 1). He was also presented with respiratory distress at birth and required CPAP with oxygen supplementation for a few days with gradual improvement. A sepsis work-up was initiated and he was started empirical treatment with ampicillin and amikacin for 72 hours (sepsis work-up was negative for infection).

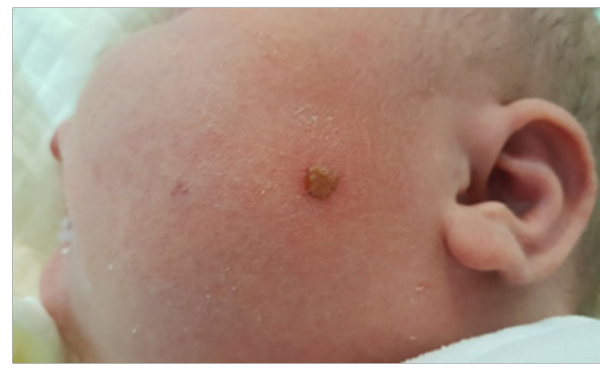

Figure I Solitary skin lesion - brown, crusted papule on left cheek.
Maternal and family history was negative for syndromes or systematic diseases and with an uncomplicated prenatal course in a provintial hospital. On clinical examination was found large for gestational age neonate without pathological reason for that. Imaging and laboratory studies were performed, and were within normal limits. On the 4th day of life a biopsy from pediatric surgeons was performed by obtaining a specimen from the papule on the left cheek and sent for histopathological examination. The specimen measured $0.5 \times 0.5 \times 0.3 \mathrm{~cm}$. Hematoxylin and eosin stained sections showed a diffuse cutaneous infiltration by medium-sized to large cells with abundant cytoplasm and characteristic irregular, elongated or with prominent folds and grooves (Figure 2A). Immunohistochemistry was performed and the cells were positive to S-100 protein (Figure 2B), CD1a antigen (Figure 2C) CD68 and HLA-DR. The diagnosis of Langerhans cell histiocytosis was set.

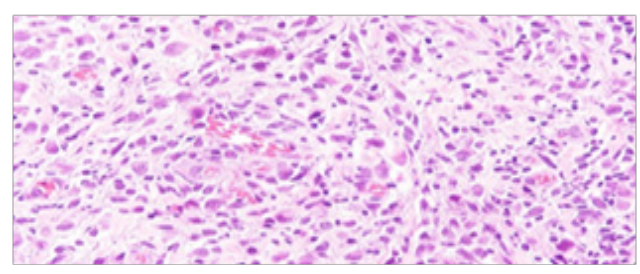

Figure 2A Hematoxylin and eosin stained sections showed a diffuse cutaneous infiltration by medium- sized to large cells with abundant cytoplasm and characteristic irregular, elongated or with prominent folds and grooves nuclei.

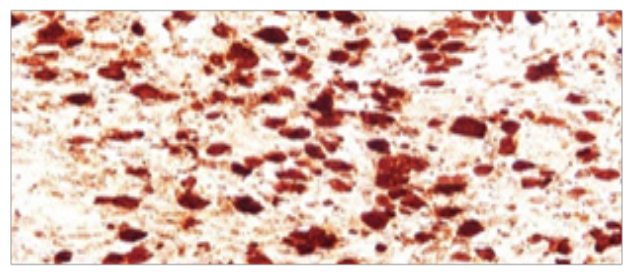

Figure 2B The cells were positive to S-100 protein. 


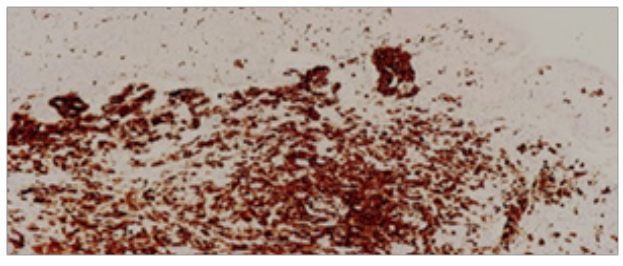

Figure 2C The cells were positive CDIa antigen.

The single skin lesion involutes spontaneously and on the second week of life, disappeared completely. The infant showed a clinical improvement over the next few days and he was discharged from the NICU department. After that he was referred to a pediatric oncology institution according our consultation

\section{Discussion}

$\mathrm{LCH}$ is the most common disorder of the large group of histiocytosis syndromes and is characterized by a monoclonal proliferation and infiltration of cells with immune phenotypic and ultra structural characteristics with Langerhans cells. ${ }^{1,4-6}$

It is more common in the male gender relative to the female. LCH has variable clinical presentation and possible outcome, can be manifested by one organ participation (skin) or as multi-organ disease, resulting in relative organ dysfunction. ${ }^{1,4,6}$

Congenital self-healingreticulohistiocytosis (CSHR) is a rare benign coutaneous form of LCH first described by Hashimoto and Pritzker (CSHR) in 1973., 2,3,7 It usually presents as multiple redbrown papules, nodules, vesicles or crusted papulovesicular lesions. ${ }^{6,8}$ developed at birth or during the neonatal period. Solitary lesions are reported in $25 \%$ of the cases. Skin findings are usually resolved spontaneously, in a few weeks or months and systemic involvement does not occur. Others rare manifestations are eczematous (seborrhealike) dermatitis, mucosal lesions, petechiae, and blueberry-muffin. ${ }^{8,9}$ Depending on multisystem involvement, congenital LCH can present as a disseminated $(50 \%)$ or a cutaneous $(50 \%)$ disease, with the last to have a better prognosis. ${ }^{6}$

Congenital cutaneous LCH demonstrates a self-limited progression and treatment options are considered only in persistent lesions or multisystem disease. Topical steroids and nitrogen mustard are considered to be effective, especially in erythematous cutaneous lesions. Prednisone and vinblastine is a therapeutic option in multisystem disease. Although, eventual progression of cutaneous LCH to multisystem disease is rather unusual, there is an increased risk of long-term internal involvement or recurrence of cutaneous lesions. A regular follow-up is always necessary for early recognition of a possible relapse due to organ infiltrations. In our case single crusted papule lesion in the face was observed immediately after birth, suggesting fetal origin. It resolved spontaneously in the $2^{\text {nd }}$ week of life.

\section{Acknowledgments}

None.

\section{Conflict of interest}

The author declares no conflict of interest.

\section{References}

1. Antonín Cuc. Experienced and well Structured Motivated Qualificated Decision Team by Patented Principles Utility model 21532 CZ. Ortho Res Online J. 2010;2(1).

2. Antonín Cuc. On A Criminal Trace of Crippling and Often Even Lethal False Diagnostics: Lege Artis CZ. Orthop and Sport Med Op Acc J. $2018 ; 1(2)$

3. Antonín Cuc. The Utility model 21532 . The Equipment for Search and Retrieval of sufficient information to reuse for Mass repeated similar strategic Decision making with risk and Computer support. Czech Office for Industrial Law, Prague, CZ; 2010. 